\title{
Ultraviolet Photochemistry of Some Frozen Gases
}

\author{
W ARREN E. THOMPSON \\ Case Institute of Technology \\ Cleveland, Ohio
}

$\mathrm{T}$

HERE SEEMS TO BE AGREEMENT that laboratory studies are necessary in the pursuit of a better understanding of the interstellar medium. Herein is described some experiments performed on the effects of far ultraviolet radiation on molecules containing the astronomically abundant atoms $\mathrm{H}, \mathrm{O}, \mathrm{C}$, and $\mathrm{N}$. In particular, the attempts that have been made to prepare, isolate, and identify various free radicals and other reactive species, some of which may play a role in the processes occurring in the interstellar medium, are discussed.

\section{CARBON TRIOXIDE}

In one of the studies, a frozen film of pure carbon dioxide at $77^{\circ} \mathrm{K}$ was irradiated with the light from a low pressure xenon discharge lamp equipped with a $\mathrm{LiF}$ ' window. This lamp is activated by radio-frequency radiation, and the emitted light is rich in the $1470-\AA$ resonance line of xenon. A comparison of the infrared absorption spectrum of the $\mathrm{CO}_{2}$ film before and after irradiation showed that several new bands appeared as a result of the irradiation. One of these, at $2139 \mathrm{~cm}^{-1}$, is attributed to $\mathrm{CO}$, presumably produced along with an oxygen atom in the primary photolysis step:

$$
\mathrm{CO}_{2} \stackrel{n v}{\rightarrow} \mathrm{CO}+\mathrm{O}
$$

where $h$ is Planck's constant and $\nu$ is frequency. The other new features, at $2045 \mathrm{~cm}^{-1}, 1880 \mathrm{~cm}^{-1}, 1073 \mathrm{~cm}^{-1}$, and $972 \mathrm{~cm}^{-1}$, could not be assigned to known molecules. This was evidence that some unknown molecular species was being produced in the solid $\mathrm{CO}_{2}$. Further infrared spectroscopic studies supported the conclusion that the species contains just one carbon atom and very likely three oxygen atoms. This conclusion was reached as a result of performing photolytic studies on carbon dioxide enriched in $\mathrm{C}^{13}$ and in $\mathrm{O}^{18}$. For example, when a solid film of carbon dioxide consisting of 56 percent $\mathrm{C}^{13} \mathrm{O}_{2}$ and 44 per- 
cent $\mathrm{C}^{12} \mathrm{O}_{2}$ was irradiated, the resulting infrared spectrum showed that three of the previously mentioned bands had lower frequency counterparts of about the same intensity. The fourth band, at 1073 $\mathbf{c m}^{-1}$, was relatively twice as intense as before, indicating that it was composed of two unresolved bands. From these results it was concluded that the new molecule possesses just one carbon atom. The molecules containing $\mathrm{C}^{12}$ gave rise to the set of higher frequency bands, and those with $\mathrm{C}^{13}$, to the lower frequency set. If more than one carbon atom were present in the molecule, the resulting larger number of possible isotopic species would have yielded a more complex spectrum. In a similar way it was concluded from experiments utilizing $\mathrm{CO}_{2}$ enriched to varying degrees in $\mathrm{O}^{18}$ that there are three oxygen atoms present in the molecule.

One can write various structures for this carbon trioxide molecule, and at the present time attempts are being made to determine which one is correct. This determination involves finding all the infraredactive fundamentals, considering their frequencies, and measuring the frequency shifts upon isotopic substitution. For $\mathrm{CO}_{3}$, this work is still in progress.

The visible-ultraviolet spectrum of $\mathrm{CO}_{3}$ is of interest to astronomers. Unfortunately, attempts to record this spectrum have been unsuccessful to date. The molecule is easily decomposed photolytically by light in both the near and far ultraviolet.

\section{PHOTOLYSIS UF CARBON SUBOXIDE}

In a second study, carbon suboxide, $\mathrm{C}_{3} \mathrm{O}_{2}$, was embedded in a solid argon matrix at $4^{\circ} \mathrm{K}$ in the ratio of one part $\mathrm{C}_{3} \mathrm{O}_{2}$ to 700 parts argon. The deposit was then irradiated by using the previously mentioned xenon discharge lamp. The purpose of this experiment was (if possible) to produce and isolate the $\mathrm{CCO}$ radical:

$$
\mathrm{OCCCO} \stackrel{h \nu}{\rightarrow} \mathrm{CCO}+\mathrm{CO}
$$

Several new infrared absorption features appeared, including three near $381 \mathrm{~cm}^{-1}, 1074 \mathrm{~cm}^{-1}$, and $1978 \mathrm{~cm}^{-1}$. In addition, the strong absorption band of $\mathrm{CO}$ at $2140 \mathrm{~cm}^{-1}$ was observed. At the same time that we were doing these experiments, M. E. Jacox and D. E. Milligan were carrying out studies on the photolysis of cyanogen azide, $\mathrm{N}_{3} \mathrm{CN}$. They found that in the presence of $\mathrm{CO}$, photolysis of $\mathrm{N}_{3} \mathrm{CN}$ leads to the same three bands that we observed. Further work by Jacox and Milligan that involved isotopic substitution with $\mathrm{C}^{13}, \mathrm{~N}^{15}$, and $\mathrm{O}^{18}$ showed that the absorption features indeed arise from the linear molecule $\mathrm{CCO}$, and that in their study it had been produced by the reaction of carbon atoms with $\mathrm{CO}$. One interesting feature of this molecule is its reactivity with carbon monoxide, even at temperatures as low as 
$4^{\circ} \mathrm{K}$, to form carbon suboxide, $\mathrm{C}_{3} \mathrm{O}_{2}$. Apparently there is very little or no activation energy required for this reaction.

Jacox and Milligan, in a study of the $7000 \AA$ to $2500 \AA$ spectral region, found that CCO shows a broad absorption near $5000 \AA$.

\section{NCN}

If carbon suboxide is embedded in a matrix of solid nitrogen rather than solid argon, and the deposit is irradiated as before, one again obtains the spectroscopic features characteristic of $\mathrm{CCO}$, and some new bands as well. The new bands were puzzling at first because they apparently arise as a result of reaction with the $\mathrm{N}_{2}$ matrix, and $\mathrm{N}_{2}$ was thought to be essentially inert at $4^{\circ} \mathrm{K}$. Two of these new bands, at 423 $\mathrm{cm}^{-1}$ and $1478 \mathrm{~cm}^{-1}$, agree in wave number with spectroscopic features arising from the photolysis of $\mathrm{N}_{3} \mathrm{CN}$, studied in detail as reported in reference 1 . These features were shown by them to arise from the molecular species NCN. This is strong evidence that in our experiment carbon atoms, produced by the photolysis of CCO, reacted with $\mathrm{N}_{2}$ by insertion to form NCN. It is possible that other infrared absorption features observed in this experiment arise from CNN, formed by terminal addition of carbon to $\mathrm{N}_{\mathbf{2}}$.

\section{CARBON ATOM REACTIONS}

In the foregoing discussion evidence has been presented that indicates that photolytically produced carbon atoms react with both $\mathrm{CO}$ and $\mathrm{N}_{2}$. It seemed of interest to see whether carbon atoms produced in this way would react with other simple molecules, such as $\mathrm{H}_{2}, \mathrm{NO}$, and $\mathrm{HCl}$. When a deposit of $\mathrm{C}_{3} \mathrm{O}_{2}, \mathrm{H}_{2}$, and $\mathrm{Ar}$ is irradiated with ultraviolet light, as described previously, several new infrared absorption bands appear in the spectrum. Most easily identified are methane $\left(\mathrm{CH}_{4}\right)$ and ketene $\left(\mathrm{CH}_{2} \mathrm{CO}\right)$. The replacement of hydrogen in the matrix by deuterium leads to the formation of $\mathrm{CD}_{4}$ and $\mathrm{CD}_{2} \mathrm{CO}$; and when a mixture of $\mathrm{H}_{2}$ and $\mathrm{D}_{2}$ is used, $\mathrm{CH}_{2} \mathrm{CO}, \mathrm{CD}_{2} \mathrm{CO}, \mathrm{CH}_{4}, \mathrm{CD}_{4}$, and $\mathrm{CH}_{2} \mathrm{D}_{2}$ are produced. These products suggest that methylene $\left(\mathrm{CH}_{2}\right.$ or its deuterium counterpart) is produced by the reaction of carbon atoms with $\mathrm{H}_{2}$ :

$$
\mathrm{C}+\mathrm{H}_{2} \rightarrow \mathrm{CH}_{2}
$$

The methylene thus produced then reacts with a nearby molecule of $\mathrm{H}_{2}$ or $\mathrm{CO}$ (the latter also being produced in the photolysis of $\mathrm{C}_{3} \mathrm{O}_{2}$ and $\mathrm{CCO})$ :

$$
\begin{aligned}
\mathrm{CH}_{2}+\mathrm{H}_{2} & \rightarrow \mathrm{CH}_{4} \\
\mathrm{CH}_{2}+\mathrm{D}_{2} & \rightarrow \mathrm{CH}_{2} \mathrm{D}_{2} \\
\mathrm{CH}_{2}+\mathrm{CO} & \rightarrow \mathrm{CH}_{2} \dot{\mathrm{CO}}
\end{aligned}
$$


Experiments designed to trap methylene before it could react further were unsuccessful. Methylene is known to be very reactive, and in these experiments it was generated in the vicinity of molecules of $\mathrm{CO}$ and $\mathrm{H}_{2}$, for which it apparently has great affinity.

It would be incorrect to conclude from these experiments that the carbon atoms reacting with $\mathrm{CO}, \mathrm{N}_{2}$, and $\mathrm{H}_{2}$ have electronic and kinetic energies corresponding to $4^{\circ} \mathrm{K}$, the temperature of the matrix. Depending on the energy of the photon responsible for the process, the liberated carbon atom may be in any of several electronic states. Furthermore, it will in general be produced with excess kinetic energy, and may well react with some environmental molecule before losing this energy by nonreactive collisions. Further studies are needed to determine the required activation energies for these reactions.

\section{ULTRAVIOLET-VISIBLE SPECTRA AND MATRIX EFFECTS}

With our own experimental apparatus it has not generally been possible to record the ultraviolet-visible spectra of the product of photolysis. Such spectra are, of course, of importance to astronomers, and fortunately, there are workers in this field who are presently equipped to record such spectra. One point that should be mentioned is that the appearance and frequency of a spectral feature arising from an embedded molecule often depend on the composition and physical condition of the surrounding matrix.

\section{SUMMARY}

Various low-temperature experiments in which chemical reactions are induced in various pure and mixed frozen gases by vacuum ultraviolet irradiation have been discussed. Experimental evidence for the formation of $\mathrm{CO}_{3}, \mathrm{CCO}, \mathrm{NCN}$, and $\mathrm{HCH}$ has been presented, and the usefulness of infrared spectroscopy in identifying such species and in determining their structures has been illustrated. These studies have demonstrated the reactivity of photolytically produced carbon atoms toward $\mathrm{CO}, \mathrm{N}_{2}$, and $\mathrm{H}_{2}$. There is a need for further ultraviolet-visible studies, including studies with matrices appropriate to the astronom. ical problem.

\section{REFERENCES}

1. Milligan, D. E.; Jacox, M. E.; Comeford, J. J.; and ManN, D. E.: Infrared Spectrum of the Free Radical NCN. J. Chem. Phys., vol. 43, 1965, pp. 756-757.

The following papers contain additional studies on this subject:

2. Jacox, M. E.; Milligan, D. E.; Moll, N. G.; and Thompson, W. E.: Matrix-Isolation Infrared Spectrum of the Free Radical CCO. J. Chem. Phys., vol, 43, 1965, pp. 3734-3746.

3. Milligan, D. D.; Jacox, M. E.; and Bass, A. M.; Matrix Isolation Study of the Photolysis of Cyanogen Oxide. The Infrared and Ultraviolet Spectra of the Free Radical NCN. J. Chem. Phys., vol. 43, 1965, p. 3149. 
4. Moll, N. G.; and Thompson, W. E.: Reactions of Carbon Atoms with $\mathrm{N}_{2}, \mathrm{H}_{2}$ and $\mathrm{D}_{2}$ at $4.2^{\circ}$ K. J. Chem. Phys., vol. 44, 1966, p. 2684.

5. Moll, N. G.; Clutter, D. R.; and Thompson, W. E.: Carbon Trioxide: Its Production, Infrared Spectrum, and Structure Studied in a Matrix of Solid $\mathrm{CO}_{2}$. J. Chem. Phys., vol. 45,1966 , pp. $4469-4481$.

\section{DISCUSSION}

Harteck: Did you say that the $\mathrm{C}_{2} \mathrm{O}$ radical was stable, or did it decompose when it was warm, or was it very fast reacting?

W. E. Thompson: It reacts very fast with carbon monoxide. Over a period of minutes it disappears from the spectrum. It reacts quickly with $\mathrm{CO}$ to form carbon suboxide at $4^{\circ} \mathrm{K}$.

Harteck: But this is very difficult to do and isn't very likely.

W. E. Thompson: You can photolyze $\mathrm{C}_{2} \mathrm{O}$ away eventually to get carbon atoms, and carbon monoxide.

Harteck: Yes, I know. I am just saying that at a low temperature $\mathrm{C}_{2} \mathrm{O}$ is stable.

W. E. Thompson: If you warm up the matrix to a sufficiently high temperature so that diffusion can occur, then it will react.

Harteck: Then it is gone.

W. E. Thompson: Not quantitatively gone; the bands decrease, perhaps to 20 percent of their original intensity.

Harteck: Do you really think these reactions will occur at $0^{\circ}$ ?

W. E. Thompson: I wouldn't be surprised if $\mathrm{C}_{2} \mathrm{O}$ reacted with $\mathrm{CO}$ within $2^{\circ}$ of absolute zero. What I am surprised about is our evidence that carbon atoms react with $\mathrm{N}_{2}$ to form NCN. If this is correct, then the carbon atom inserts between the two nitrogen atoms, which are bound by a triple bond. My only evidence for this is the agreement with Milligan's results on the NCN radical. We get the same band. Remember that the carbon atoms might be quite hot. These carbon atoms are not at $4^{\circ} \mathrm{K}$ when they are formed. Therefore, considerable activation energy may be necessary, and it may be provided by the photolytic process. In the photolysis of the $\mathrm{C}_{2} \mathrm{O}$ you may get a hot $\mathrm{C}$ atom which can then hit a nitrogen molecule.

Harteck: I don't know all the details, but it is known that if you make $\mathrm{C}$ atoms photochemically, they enter the molecules.

W. E. Thompson: Yes, I am aware of that.

Harteck: And so they may enter the nitrogen.

W. E. Thompson: To enter a triple bond is quite a feat.

Harteck: That is why I asked you; but the whole of the reaction is exothermic.

W. E. Thompson: I'm glad to hear you say that. We have made the same calculation. I was worried more about the activation energy that was required. 